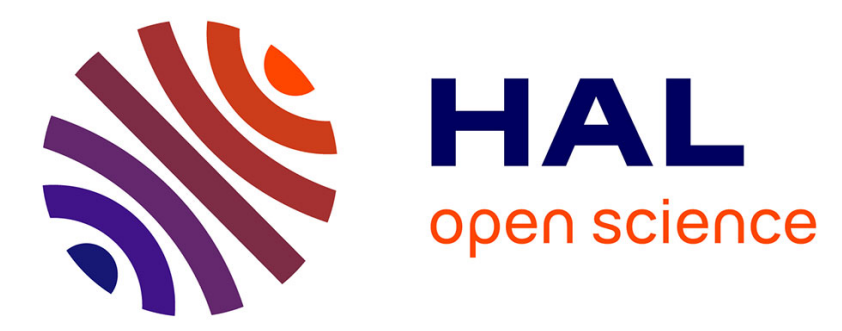

\title{
Le bien commun dans la littérature anglaise de la fin du Moyen Âge \\ Aude Mairey
}

\section{To cite this version:}

Aude Mairey. Le bien commun dans la littérature anglaise de la fin du Moyen Âge. Revue Française d'Histoire des Idées Politiques, 2010, Pouvoir d'un seul et bien commun, Ve-XVIe siècle, Actes du colloque international de Nanterre (décembre 2008), 32, pp.373-384. halshs-01078922

\section{HAL Id: halshs-01078922 \\ https://shs.hal.science/halshs-01078922}

Submitted on 25 Nov 2015

HAL is a multi-disciplinary open access archive for the deposit and dissemination of scientific research documents, whether they are published or not. The documents may come from teaching and research institutions in France or abroad, or from public or private research centers.
L'archive ouverte pluridisciplinaire HAL, est destinée au dépôt et à la diffusion de documents scientifiques de niveau recherche, publiés ou non, émanant des établissements d'enseignement et de recherche français ou étrangers, des laboratoires publics ou privés. 


\section{Aude Mairey (CNRS/LaMOP)}

\section{Le bien commun dans la littérature anglaise de la fin du Moyen Âge}

Pour beaucoup d'historiens et de critiques littéraires travaillant sur l'Angleterre des XIV et $\mathrm{XV}^{\mathrm{e}}$ siècles, de nombreuses sources littéraires, et en particulier toutes celles que l'on pourrait ranger sous la bannière de la poésie politique (bien que cette poésie ne soit en général pas seulement politique), insistent sur la nécessité du bien commun, à tel point que l'expression semble avoir la saveur d'un cliché éculé ${ }^{1}$ : tous ceux qui participent au gouvernement du royaume, que ce soit directement ou indirectement, sont priés de faire passer le bien commun avant leurs intérêts particuliers, de mettre leurs pouvoirs et leurs compétences variées à son service, sous la direction éclairée du roi $^{2}$. Mais force est de constater que cette expression n'apparaît que rarement en tant que telle dans les textes et, surtout, que ses formes sont plurielles : common good, common wele, common profit... En envisageant les contextes précis où apparaissent ces expressions, nous tenterons donc de mieux cerner leur signification et l'impact de leur emploi dans la constitution d'un dialogue politique entre le roi et ses sujets, élément-clé de l'histoire anglaise de la fin du Moyen Âge.

Les textes utilisés pour cette étude font partie d'un corpus que j’ai constitué pour analyser plus précisément les langages politiques anglais aux $\mathrm{XIV}^{\mathrm{e}}$ et $\mathrm{XV}^{\mathrm{e}}$ siècles. Deux thèmes ont déjà fait l'objet d'une étude appuyée sur toute ou partie de ce corpus, celui du modèle royal et celui de la notion de peuple ${ }^{3}$. Je ne présenterai donc que très brièvement les textes qui le composent, de manière typologique.

Piers Plowman, de William Langland ${ }^{4}$, et les poèmes dits de sa tradition - Richard the Redeless, Mum and the Sothsegger et The Crowned Kyng - sont des poèmes allitératifs (la répétition de consonnes et/ou de voyelles est utilisée pour donner sa cohérence au vers), ce qui les distingue

\footnotetext{
${ }^{1}$ Pour une vision assez sévère de la littérature politique de l'époque, considérant que celle-ci est très stéréotypée, voir G. Harriss, Shaping the Nation. England, 1360-1461, Oxford, 2005, p. 6-13. Cet ouvrage est par ailleurs extrêmement précieux en tant que synthèse sur l'histoire de la période.

${ }^{2}$ Sur la question des représentations du rôle des différents groupes sociaux, je me permets de renvoyer à mon article écrit en collaboration avec Franck Collard, «La société politique en France et en Angleterre au miroir de ses représentations mutuelles ", dans France et Angleterre : Deux Etats, Deux sociétés politiques, éd. J.-P. Genet et J. Watts, à paraître.

3 A. Mairey, «Les modèles royaux dans la poésie anglaise de la fin du Moyen Âge ", dans Vérité poétique, vérité politique : mythes, modèles et idéologies politiques au Moyen Age, éd. J.-C. Cassard, E. Gaucher et J. Kerhervé, Brest, 2007, p. 297-315; "Qu'est-ce que le peuple? Quelques réflexions sur la littérature politique anglaise de la fin du Moyen Âge», à paraître dans Médiévales.

${ }_{4}^{4}$ William Langland, The vision of Piers Plowman, Version B, éd. A.V.C. Schmidt, Londres, 1978, 1987 ; Pierre le laboureur, trad. A. Mairey, Paris, 1999.

5 The Piers Plowman Tradition, éd. H. Barr, Londres, 1993.
} 
formellement des autres. Piers Plowman, de loin le plus important, est d'abord un poème sur le salut de l'homme et de la société; Richard, Mum et The Crowned Kyng, s'ils sont directement influencés par Piers au point d'être qualifiés de poèmes de sa tradition, sont plus pragmatiques ${ }^{6}$. Le livre VII de la Confessio amantis de John Gower (accompagné de son prologue) ${ }^{7}$ et le Regement of Princes de Thomas Hoccleve ${ }^{8}$ sont des miroirs au prince poétiques qui recourent de façon massive à des exempla pour illustrer leur propos - donner des conseils sur le bon gouvernement ${ }^{9}$. Si le premier n'a pas été commandité, le second a probablement été commandé (ou du moins impulsé) par Henry V juste avant son accession au trône, vers 1411-1412. The Regement of Princes est donc un poème lancastrien, mais qui dépasse de loin le simple poème de circonstance. Le poème de George Ashby est également un miroir au prince ${ }^{10}$; il date de 1470 environ et a été composé pour le fils d'Henry VI (alors en exil); il ne contient pas d'exempla, seulement des conseils directs à l'héritier du royaume (qui ne règnera en fait jamais), dans la période troublée de la guerre civile ${ }^{11}$. Les poèmes du Digby $102^{12}$ sont en partie des poèmes de circonstances, écrits par un seul auteur, peut-être un clerc londonien qui connaissait bien le parlement, sur une période allant de 1400 à 1430 environ ${ }^{13}$.

Dans ces textes, l'expression précise de la notion de bien commun n'apparaît que très incidemment, comme le suggère le tableau suivant, qui regroupe les associations de l'adjectif qualificatif commune, c'est-à-dire les mots qu'il qualifie :

\footnotetext{
${ }^{6}$ Sur ces poèmes, voir A. Mairey, Une Angleterre entre rêve et réalité. Littérature et société en Angleterre au XIV siècle, Paris, 2007. ; H. Barr, Signs and Soth: language in the Piers Plowman tradition, Oxford, 1994.

7 John Gower, Confessio Amantis, vol. 1, éd. R. Peck, TEAMS, Kalamazoo, 2000 (en ligne à l'adresse suivante : http://www.lib.rochester.edu/camelot/teams/rpca1int.htm).

8 Thomas Hoccleve, The Regement of Prince, éd. C.R. Blyth, TEAMS, Kalamazoo, 1999 (en ligne à l'adresse suivante : http://www.lib.rochester.edu/camelot/teams/hoccfrm.htm). Une traduction du prologue (qui occupe plus du tiers du poème) est à paraître dans le recueil Dialogues et résistances : une anthologie de textes anglais de la fin du Moyen Áge, trad. Aude Mairey et Stephen Morrison.

${ }^{9}$ Sur ces questions, voir notamment J. Ferster, Fictions of Advice: The Literature and Politics of Counsel in Late Medieval England, Philadelphie, 1996 ; L. Scanlon, Narrative, Authority and Power: The Medieval Exemplum and Chaucerian Tradition, Cambridge, 1994.

${ }^{10}$ Georges Ashby, The Active Policy of a Prince, éd. M. Bateson, Early English Texts Society, e.s. 76, Londres, 1899.

${ }^{11}$ Cf. R. J. Meyer-Lee, "Laureates and beggars in Fifteenth-century English Poetry : the case of George Ashby", Speculum 79/3, 2004, p. 687-726.

12 Twenty-Six Political and Other Poems (Digby 102), éd. J. Kail, EETS, o.s. 124, Londres, 1904.

13 J'avais également inclus dans mon corpus des poèmes yorkistes datant de la seconde moitié du XVe siècle et composés en faveur de la dynastie yorkiste (qui a écarté la dynastie lancastrienne). Ce sont des poèmes de circonstances, mais aucune occurrence concernant le bien commun n’apparait. Ces textes sont édités dans Historical Poems of the XIVth and XVth centuries, éd. R.H. Robbins, New York, 1959.
} 
Tableau 1 - Les mots qualifiés par l'adjectif commune

\begin{tabular}{|c|c|c|c|c|c|}
\hline $\begin{array}{c}\text { Langland } \\
\left(17^{14}\right)\end{array}$ & Gower (36) & Allitér. (8) & Digby (7) & $\begin{array}{c}\text { Hoccleve } \\
(10)\end{array}$ & Ashby (3) \\
\hline $\begin{array}{l}\text { peple } 4 \\
\text { woman/men } \\
4 \\
\text { profit } 3 \\
\text { court } \\
\text { foode } \\
\text { laborers } \\
\text { lawe } \\
\text { lif } \\
\text { men }\end{array}$ & $\begin{array}{l}\text { peple 7 } \\
\text { profit } 7 \\
\text { right } \mathbf{5} \\
\text { lawe } 3 \\
\text { vois } \mathbf{3} \\
\text { clamour } \mathbf{2} \\
\text { fere } \mathbf{2} \\
\text { good } \mathbf{2} \\
\text { assent } \\
\text { counseil } \\
\text { drede } \\
\text { strif } \\
\text { wronges }\end{array}$ & $\begin{array}{l}\text { peple 2 } \\
\text { clamour } \\
\text { counseil } \\
\text { profit } \\
\text { speche } \\
\text { tale } \\
\text { wele }\end{array}$ & $\begin{array}{l}\text { vois } 3 \\
\text { lawe } 2 \\
\text { noise } \\
\text { profit }\end{array}$ & $\begin{array}{l}\text { profit } 4 \\
\text { cost } \\
\text { goodes } \\
\text { lawe } \\
\text { peple } \\
\text { treson } \\
\text { vois }\end{array}$ & $\begin{array}{l}\text { commune } \\
\text { sawe } \\
\text { wele }\end{array}$ \\
\hline
\end{tabular}

Au total, common profit apparaît 16 fois sur l'ensemble du corpus, essentiellement dans les poèmes de Gower et d'Hoccleve, common good n'apparaît que deux fois, chez Gower, de même que common wele dans Mum and the Sothsegger et chez Ashby. Il y a donc une nette domination de l'expression common profit, d'autant que Hoccleve emploie également à deux reprises l'expression universel profit. Cette faible fréquence totale n'est pas spécifique au corpus envisagé. Si l'on jette un œil aux traités politiques, on s'aperçoit qu'il en est de même; je ne prendrai que l'exemple du plus célèbre d'entre eux, The Governance of England de Sir John Fortescue ${ }^{15}$. Dans ce texte, common wele n'apparaît qu'une fois, au chapitre 12, dans le contexte de la révolte des hussites en Bohème (cette révolte était bien connue en Angleterre du fait de ses liens avec l'hérésie lollarde, seule hérésie anglaise importante de la période ${ }^{16}$ ). L'expression publike gode apparait également une fois, au chapitre 14, qui évoque la nécessité d’un bien suffisant pour le roi ainsi que l'importance du conseil pour vérifier que la supériorité du bien public sur le bien particulier est respecté, thématique qui se retrouve dans nos poèmes. Cependant, un autre traité, beaucoup moins connu, compte beaucoup plus d'occurrences de common wele. Il s'agit du Somnium Vigilantis ${ }^{17}$, un traité composé en 1459, dans le contexte de la lutte appelée plus tard guerre des roses, entre les lancastriens (le roi Henry VI en place depuis 1422, était incapable de gouverner) et les yorkistes (le duc Richard d'York a réclamé le trône en 1460 mais c'est son fils Edouard IV qui l'a récupéré en 1461) ${ }^{18}$. Ce traité présente l'intérêt de recenser les arguments des yorkistes et la réponse

\footnotetext{
$14 \mathrm{Il}$ s'agit du nombre d'occurrences totales de l'adjectif commune dans le texte.

15 The governance of England otherwise called The difference between an absolute and a limited monarchy by Sir John Fortescue, éd. Charles Plummer, Londres, Oxford University Press, 1885, réimp. New York, 1999..

${ }^{16}$ Sur l'hérésie lollarde, voir A. Hudson, The Premature Reformation, Oxford, 1988 ; sur les hussites, voir F. Šmahel, La Révolution hussite, une anomalie bistorique, Paris, 1985.

17 «A Defense of the Proscription of the Yorkists in 1459 », éd. J. P. Gilson, English Historical Review 26, 1911, p. 512525.

18 Voir, entre autre, A. F. Pollard éd., The Wars of the Roses, Londres, 1995.
} 
lancastrienne de l'auteur ${ }^{19}$. Or, une partie de ces arguments est centrée sur le bien commun. Le duc Richard d'York, en effet, en avait fait un cheval de bataille dans ses déclarations ${ }^{20}$. La thématique du bien commun apparait donc bien dans les troubles politiques, en tout cas au milieu du $\mathrm{XV}^{\mathrm{e}}$ siècle, ce qui rend d'autant plus intéressant le fait qu'elle n'apparaisse que rarement en tant que tel dans les sources littéraires.

Malgré cette fréquence relativement faible, la question des termes employés doit être posée. Quelles sont les nuances, en effet, entre profit, good et wele? D'après le Middle English Dictionary $^{21}$, common good signifie soit une bénédiction ou un avantage général, soit la richesse ou les biens collectifs d'un peuple ou d'un pays. Mais le dictionnaire ne donne que deux exemples d'utilisation de ces expressions, tous deux du $\mathrm{XV}^{\mathrm{e}}$ siècle ${ }^{22}$. Common wele renvoie pour sa part à la prospérité ou au bien-être général, mais aucun exemple ne vient appuyer cette définition. Seule l'expression common profit est pourvoyeuse de plusieurs exemples, ce qui vient confirmer la tendance à l'œuvre dans notre corpus. Selon le Middle English Dictionary, common profit, qui apparaitt au $\mathrm{XIV}^{\mathrm{e}}$ siècle, renvoie à un avantage commun ou... au bien commun, ce qui ne nous éclaire pas beaucoup. L'étude de nos textes devrait pouvoir nous permettre d'affiner ces définitions.

Car les quelques occurrences repérées dans nos textes apparaissent le plus souvent dans un contexte intéressant pour notre propos, centré sur les rapports entre le bien commun et le pouvoir royal. Cela est évident, tout d'abord, dans le poème de Langland, Piers Plowman. Deux des trois occurrences de common profit apparaissent en effet dans le prologue, au sein du récit de l'exemplum des rats et des souris (prologue, vers 146-210), qui illustre les difficultés de la fondation d'un bon gouvernement monarchique présenté auparavant. La version B du texte a sans doute été écrite à la fin du règne d'Édouard III, marqué par des troubles politiques qui se sont révélés avec acuité lors du Good Parliament en $1376^{23}$. Ce parlement a attaqué un certain nombre des favoris du vieux roi et a tenté d'imposer des réformes allant dans le sens d'un plus grand contrôle du pouvoir royal ${ }^{24}$. Or, Langland semble plutôt favorable à ces réformes, même s'il reste

\footnotetext{
${ }_{19}$ Pour une étude de ce texte, voir P. Strohm, Politique : languages of Statecraft between Chancer and Shakespeare, Notre Dame, Ind., 2005, p. 12-17. Voir également l'article de Chris Fletcher dans ce volume.

${ }^{20}$ Cf. R. A. Griffiths, «Duke Richard of York's Intentions in 1450 and the Origins of the War of the Roses », dans idem, King and Country. England and Wales in the Fifteenth Century, Londres, 1991.

${ }^{21}$ Le Middle English Dictionary est en ligne à l'adresse suivante : http://quod.lib.umich.edu/m/med/.

${ }^{22}$ Les deux exemples sont tirés respectivement du Pilgrimage of the Life of Man de John Lydgate (1430) et d'une traduction du Secretum Secretorum effectuée vers 1450.

${ }^{23}$ Cf. G. Harriss, Shaping the Nation, op. cit., par 441-444 ; G. Holmes, The Good Parliament, Oxford, 1975.

${ }^{24}$ Sur l'esprit réformateur qui paraît alors avoir soufflé, voir S. Justice et K. Kerby-Fulton, « Reformist Intellectual Culture in the English and Irish Civil Service : the Modus Tenendi Parliamentum and its Literary Relations ", Traditio 53, 1998, p. 149-202.
} 
volontairement ambivalent ${ }^{25}$. La fable des rats, des souris et du chat, est d'ailleurs marquée par cette ambiguité ${ }^{26}$. La trame est la suivante : les souris se rassemblent pour discuter de la manière dont elles pourraient se débarrasser de la tyrannie du chat. Un rat propose alors de lui passer au cou un collier garni de cloches pour pouvoir suivre tous ses mouvements. Mais les rongeurs s'aperçoivent rapidement qu'il leur sera difficile d'attacher le collier au cou du chat. Une souris conseille alors de ne pas se mêler des affaires du chat et de ne pas le provoquer - d'autant que se débarrasser de ce chat là n'empêchera pas que d'autres suivront. A priori, donc, cet exemplum suggère que l'action des communes serait inutile, voir nuisible ${ }^{27}$. Pourtant, plusieurs éléments suggèrent le contraire. D'une part, cet exemplum apparait dans le sermon que l'évêque réformateur Thomas Brinton a justement prononcé... lors du bon parlement ${ }^{28}$. D'autre part, l'expression common profit, qui est toujours positive, apparaît lorsque les souris se réunissent (vers 146-151), ainsi que dans le discours du rat :

And right so, quod that raton, reson me sheweth To bugge a belle of bras or of bright silver

And knytten it on a cokr for oure commune profit And hangen it upon the cattes hals - thanne here we mowen

Wher he ryt or rest or rometh to pleye ;

And if hym list for to laike, thanne loke we mowen And peeren in his presence the while hym pleye liketh, And if hym wratheth, be war and his wey shonye
Et donc, dit le rat, la raison m'apprend qu'il faut acheter une cloche de cuivre ou d'argent brillant, l'attacher à un collier et la pendre autour du cou du chat en vue de notre bien commun. Alors, nous saurons quand il bouge, quand il se repose, ou quand il prend son plaisir au dehors ; et s'il est d'humeur joueuse, nous le verrons bien, et nous pourrons nous montrer, et s'il est en colère, nous pourrons faire attention et nous écarter de son chemin

vers $167-175$

Elisabeth Orsten note également que l'intervention de la souris est hautement suspecte, tant dans sa construction que dans les termes employés, qui soulignent son exagération ${ }^{29}$ :

Though we hadde ykilled the cat, yet sholde ther come another

To cracchen us and al oure kynde, though we cropen under benches. $[\ldots]$

For may no renk ther reste have for ratons by nyghte.

For many mennes malt we mees wolde destruye,

And also ye route of ratons rende mennes clothes,

Nere the cat of the court that kan you overlepe ;

For hadde ye rattes youre raik ye kouthe noght rule yowselve.

I seye for me, quod the mous, I se so muchel after,

Shal nevere the cat ne the kiton by my counseil be
Même si nous devions tuer le chat, un autre apparaîtrait à sa place, pour nous étriper tous, bien que nous nous soyons cachés sous les sièges. [...]

Car, voyez-vous, les rats deviendraient si actifs pendant la nuit, que personne ne pourrait fermer l'oeil ; nous, les souris, dévorerions le grain des hommes, pendant que les rats déchireraient leurs habits, si nous n'étions retenus par la peur que le chat de la cour ne fonde sur nous soudainement; vous les rats, laissés à vous-mêmes, seriez bien incapables de vous gouverner.

Vers $185-186$ et $196-203$

\footnotetext{
${ }_{25}$ Pour les conceptions générales de Langland sur le gouvernement et le pouvoir royal, voir A. Mairey, Une Angleterre entre rêve et réalité, op. cit., p. 125 sq.

26 Pour un tableau de la présence fréquente de cette fable venant d'Orient dans les sources occidentales, voir P. F. Baum, "The Fable of belling the cat», dans Proverbia in Fabula, Essays on the Relationship of the Proverbs and the Fable, P. Carnes éd., Berne, 1988, p. 37-46. Pour le contexte anglais, voir E. Orsten, « The Ambiguities in Langland's Rat Parliament », Mediaeval Studies 23, 1961, p. 216-239.

27 C'est notamment l'interprétation donnée par Anna Baldwin dans son ouvrage The Theme of Government in Piers Plowman, Cambridge, 1981, p. 17.

28 The Sermons of Thomas Brinton, Bishop of Rochester (1373-1389), éd. M.A. Devlin, 2 vol., Londres, 1954, ii, p. $316-321$. Pour les circonstances de ce sermon, voir Holmes, The Good Parliament, op. cit., p. 103-104.

${ }^{29}$ E. Orsten, «The Ambiguities in Langland's Rat Parliament », op. cit., p. 238.
} 
greved.

L'apparition, à deux reprises dans cet exemplum, de l'expression common profit, nous parait donc un indicateur fort de sa signification pour Langland, dans la mesure où il la fait intervenir à propos d'un questionnement sur la tyrannie.

En outre, le rat est mené par la raison, autre élément positif chez Langland. Et il faut souligner que la troisième occurrence de common profit apparait dans la bouche du personnage allégorique de Raison qui, dans un discours prononcé à la cour du roi contre des personnages peu recommandables (Wrong et Mede, qui incarnent la corruption), dénonce le mauvais comportement de toutes les couches de la société et en appelle à sa réforme. C'est dans ce cadre qu'il insiste sur le fait que le conseil du roi devrait s'occuper du bien commun :

Reed me noght, quod Reson, no ruthe to have Til lordes and ladies loven alle truthe And haten alle harlotrie, to heren or to mouthen it ; $[\ldots]$ Til the Kynges counseil be the commune profit ; Til bisshopes bayardes ben beggeris chaumbres...
Ne me dites pas que je devrais avoir pitié, répondit Raison, aussi longtemps que les seigneurs et leurs dames ne se seront pas tous voués à une honnête vie, haïssant toute obscénité à entendre ou à prononcer. [...]

Les avis du conseil du Roi viseront au bien commun. Les évêques, au lieu d'acheter des chevaux de luxe, utiliseront leur argent à loger les mendiants sans logis... vers $113-115$ et $124-125$.

Cette occurrence renvoie à une thématique un peu différente de la précédente, mais qui lui est liée. Il s'agit de la thématique du conseil, extrêmement importante dans la poésie anglaise de l'époque - et cela ne concerne pas seulement le conseil royal mais aussi le conseil en général, qui doit être donné au roi par les membres de la communauté ${ }^{30}$. Or, le bien commun apparaît en relation avec cette thématique dans trois des textes de notre corpus. Dans Mum and the Sothsegger, common wele est utilisée à propos du conseil royal, mené par des hommes un peu trop avides :

For thegre enuye that eche had to other

Dide thaym preece to be pryvy and put aweye the beste, But muche more for the mede to make thaym-self riche Thenne to cunseille the king of the comune wele, Or for any deue dome or defence of the royaulme.
Car l'envie avide des uns par rapport aux autres les ont fait se presser d'être secrets et de mettre le meilleur de côté ; mais [ce fut] bien plus pour la récompense de devenir eux-mêmes riches que pour conseiller le roi sur le bien commun, pour juger de manière appropriée ou pour défendre le royaume.

vers $1657-1661$

Dans un des poèmes du manuscrit Digby 102, Truth, Rest and Peace, il apparait en relation avec le parlement; selon l'auteur en effet, «Pour savoir si le parlement est sage, [il faut voir] s'il met en avant le bien commun $»^{31}$. Enfin, dans le poème d'Ashby, common wele est relié à la nécessité pour le roi de mener des enquêtes - ce qui apparaît bien comme une manière de prendre conseil auprès de tous :

If I shal speke of the vniuersal

Si je dois parler du bien commun et universel dans ce

\footnotetext{
30 Voir J.-P. Genet, «De Richard II à Richard III : le Conseil », dans «A l'ombre du pouvoir. Les entourages princiers au Moyen Âge, éd. A. Marchandisse et J.-L. Kupper, Genève, 2003, p. 177-202 et A. Mairey, Une Angleterre entre rêve et réalité, op. cit., p. 154 sq.

${ }^{31}$ Vers 97-98: To wete yif parlement be wys,/ The comoun profit wel it preues.
} 
And the comyn wele of this region, I wol aduise you in especial

To haue goode guidyng and inspeccion

To euery trouble in this nacion,

For thaugh by a litil it begynnyth,

It may distroy vs al or it endithe. pays, je vous conseillerai particulièrement d'avoir un bon conseil et d'inspecter chaque trouble de cette nation, car ce qui peut commencer petitement, peut finalement tous nous détruire.

vers $778-784$

Ces exemples suggèrent donc un lien fort entre la thématique du bien commun et la question du gouvernement du roi et de son conseil, ainsi que sur ses glissements possibles vers ses excès, à commencer par le plus grand d'entre eux, la tyrannie.

Bien que de manière un peu différente, Gower aborde les mêmes questions. Cette différence d'approche est liée à la nature même de son texte, car le livre VII de la Confessio amantis est un miroir au prince poétique; mais elle tient aussi à l'influence d'une tradition plus «aristotélicienne» que chez Langland. Quoi qu’il en soit, Gower est le poète qui utilise l'expression le plus fréquemment. Et il faut souligner que ses œuvres sont également marquées par des accents réformateurs ${ }^{32}$. Selon ses critiques, d'ailleurs, il se serait davantage inspiré du Trésor de Brunetto Latini, composé par un florentin attaché au gouvernement communal ${ }^{33}$, que du De regimine principum de Gilles de Rome, dont les accents monarchiques ne sont plus à présenter. Cette hypothèse est d'autant plus plausible que le Trésor - ou du moins certaines de ses parties - était connu à Londres depuis le début du XIV siècle grâce, notamment, à Andrew Horn, chambellan de la cité entre 1320 et $1328^{34}$. Cela ne signifie pas, bien sûr, que Gower rejettait la forme monarchique, simplement qu'il était particulièrement sensible à la question des limites.

De fait, c'est dans un exemplum consacré à une réflexion sur la nature de l'office royal en relation avec l'établissement des lois que common profit apparait à trois reprises. Il s'agit de l'histoire de Lycurgue, qui apparaît chez Gower comme un roi athénien et non comme un législateur spartiate $^{35}$. Lycurgue donne des lois aux Athéniens puis leur annonce son départ et leur fait jurer qu'ils ne changeront pas ses lois en l'attendant. Mais Lycurgue décide de ne jamais revenir, ce qui oblige les Athéniens à conserver ses lois pour toujours. Pour Gower, contrairement à d'autres auteurs, cette décision est délibérée. La ruse de Lycurgue constituerait à la fois une forme de

\footnotetext{
32 Même si sa conception de la communauté est sans doute plus restreinte que celle de Langland : voir A. Mairey, «Qu'est-ce que le peuple? », art. cité.

${ }^{33}$ Li Livres du Tresor, éd. F. J. Camody, Berkeley, 1939-1948, réimp. Genève, 1975.

${ }^{34}$ Cf. J. Simpson, Reform and Cultural Revolution. The Oxford English Literary History, vol. 2, 1350-1547, Oxford, 2002, p. 219-223 ; L. Staley, Languages of power in the age of Richard II, University Park, 2005, p. 32-39. Sur Andrew Horn et sa diffusion du Trésor, voir J. Catto, "Andrew Horn : Law and History in Fourteenth-Century England», dans The Writing of History in the Middle Ages: Essays presented to R. W. Southern, éd. R. H. C. Davis, J. M. Wallace-Hadril et alii, Oxford, 1981, p. 367-392.

${ }^{35}$ Lycurgue était assez connu au Moyen Âge, par le biais des Vies de Plutarque.
} 
liberté et de contrôle suprême (ses lois restent immuables), mais le prix à payer pour le roi est élevé $e^{36}$. Dans les trois cas, common profit apparait en rapport avec la justification de l'établissement des lois : dans la bouche de Lycurgue lorsqu'il explique les raisons de son départ et demande aux athéniens de prêter serment, puis dans la conclusion de l'exemplum:

For after that he was ago,

He schop him nevere to be founde ;

So that Athenis, which was bounde,

Nevere after scholde be relessed,

Ne thilke goode lawe cessed,

Which was for comun profit set.

And in this wise he hath it knet;

He, which the comun profit soghte,

The king, his oghne astat ne roghte;

To do profit to the comune,

He tok of exil the fortune,

And lefte of Prince thilke office

Only for love and for justice...
Car après être parti, il s'arrangea pour qu'on ne le retrouve jamais afin qu'Athènes, qui était liée, ne soit jamais libérée et que les bonnes lois, établies pour le bien commun, ne soient pas abolies. Et c'est ainsi qu'il avait procédé, lui qui recherchait le bien commun. Le roi ne se souciait pas de son état: il choisit la condition de l'exil et abandonna l'office de prince pour le profit de la communauté, pour l'amour et pour la justice...

vers 3002-3014

Là encore, l'utilisation de la notion de bien commun dans un exemplum mettant l'accent sur l'idée du sacrifice du roi pour la bonne conservation de ses lois est significative de l'importance de la notion dans le cadre d'une réflexion sur le pouvoir royal et le gouvernement. La mise en relation entre le bien commun et la loi apparait d'ailleurs dans la conclusion d'un autre exemplum, qui se situe également dans la partie sur la justice et qui raconte une anecdote sur le consul romain Gaius Fabricius ${ }^{37}$ :

To sette a lawe and kepe it noght

Ther is no comun profit soght; Bot above alle natheles The lawe, which is mad for pes, Is good to kepe for the beste, For that set alle men in reste.
Le bien commun ne peut être recherché si l'on instaure une loi et que l'on ne la respecte pas. Car par-dessus tout, en vérité, la loi, qui est faite pour la paix, doit être protégée pour le mieux - elle apaise les hommes.

vers $2827-2832$

Cette dernière anecdote, cependant, nous renvoie à une problématique un peu différente de la précédente, qui constitue un autre versant du bien commun, plus «matériel». Car elle raconte comment Gaius a refusé de l'or proposé par les Samnites après qu'il leur a fait accorder la paix. La raison donnée en est qu'il s'intéressait davantage à la justice, y compris envers les riches, qu'à la richesse elle-même. Cela renvoie donc à la question de la gestion des biens par le roi (les siens propres et ceux de ses sujets), qui apparaît ailleurs chez Gower, dans le contexte de la discussion sur la largesse :

The worldes good was ferst comune,

Bot afterward upon fortune

Was thilke comun profit cessed:

For whan the poeple stod encresced
Les biens de ce monde furent d'abord tenus en commun mais ensuite, de par la fortune, ce bien commun fut supprimé ; car lorsque les gens se multiplient et que les lignages s'élargissent, chaque homme est bientôt tiré

\footnotetext{
${ }^{36}$ Cf. L. Scanlon, Narrative, Authority and Power: The Medieval Exemplum and Chaucerian Tradition, Cambridge, 1994 , p. 287-289.

37 Gaius Fabricius a été consul en 282 avant J.-C. Son histoire est racontée par Valère Maxime, une des sources de Gower.
} 
And the lignages woxen grete, Anon for singulier beyete

vers son parti pour des gains privés.

Drouh every man to his partie ;

Gower remonte donc à l'origine de l'apparition de la propriété pour cadrer sa discussion sur la largesse royale, qui doit s'opposer à la cupidité bien sûr (le terme anglais, avarice, renvoie davantage à la cupidité en français), mais aussi à la prodigalité.

Dans cet exemplum, commune good renvoie clairement aux biens terrestres. C'est également le cas pour l'autre occurrence de l'expression chez Gower, qui apparait au début de l'exemplum de Lycurgue, dans le contexte de la description des effets du bon gouvernement royal : « La richesse était ordonnée pour le bien commun et non pour le particulier... $»^{38}$. En revanche, common profit est plus polysémique, même s’il semble avoir souvent un sens plus général. Mais il reste difficile de préciser exactement si, dans le cas des occurrences de common profit qui ne sont pas restreintes aux biens terrestres, la référence est plutôt à une conception générale du bien commun comme une «vie de vertu», plutôt aristotélicienne ou à une conception plutôt utilitaire renvoyant au bien-être de la société, plus augustinienne ${ }^{39}$.

Quoi qu'il en soit, c'est surtout Thomas Hoccleve qui développe la question du bien commun en relation avec la vertu de largesse et plus généralement en relation avec le thème de la prospérité. De fait, c'est le poète le plus sensible à ce type de problèmes, ce qui s'explique en partie par le fait que son poème est tout autant un miroir qu'une pétition présentée au prince pour obtenir l'assurance que sa pension continuera à être versée lorsqu'il ne pourra plus travailler comme scribe. La situation matérielle et sociale des hommes - et la manière dont le roi peut l'assurer - est un des éléments-clés de la réflexion d'Hoccleve. Dans ce cadre, ses développements sur la vertu de largesse et sur les vices de la prodigalité et de l'avarice (on notera d'ailleurs qu'il est plus indulgent envers le premier qu'envers la seconde), apparaissent particulièrement importants. Pratiquement toutes les occurrences de common profit (ou d'universel profit) sont en rapport avec ces thématiques, y compris dans le prologue ${ }^{40}$. La première occurrence apparaît en relation avec une critique de l'extravagance de la mode :

Now wolde God the waast of clooth and pryde Yput were in exyl perpetuel

For the good and profyt universel ; And lordes mighte helpe al this, if they wolde The old get take, and it foorth use and holde. Than mighte silver walke more thikke Among the peple than that it dooth now.

\begin{abstract}
Vraiment, Dieu veuille que le gâchis de tissus et l'orgueil soient mis en exil perpétuel pour le bien et le profit universels; les seigneurs pourraient aider à cela, s'ils le voulaient, en reprenant l'ancienne mode et en l'utilisant à nouveau. L'argent circulerait alors parmi les gens davantage qu'il ne le fait maintenant.
\end{abstract}

vers $521-527$

\footnotetext{
${ }^{38}$ Vers 2930-2932 : Richesse upon the comun good/ And noght upon the singuler/ Ordeigned was...

${ }^{39}$ Voir à ce sujet l'ouvrage important de Matthew Kempshall, The Common Good in late medieval political thought, Oxford, 1999.

${ }^{40}$ La seule exception concerne l'histoire de Marcus Regulus sur la fidélité à la parole donnée.
} 
La critique de l'apparence est fréquente à la fin du XIV et au début du $\mathrm{XV}^{\mathrm{e}}$ siècle, en relation avec des transformations importantes de la mode à cette époque ${ }^{41}$. Mais elle est ici particulièrement prononcée et clairement associée à la prospérité du pays. La seconde occurrence du prologue est dans la même veine. Elle apparaît en effet dans un exemplum présentant un roi sicilien d'origine modeste, utilisant de la vaisselle d'argile à sa table. Le contexte est celui d'un éloge de la mesure nécessaire pour contrer les caprices de la fortune, mais Hoccleve relie cela au bien commun - un roi devant toujours se gouverner en gardant le bien-être de ses sujets à l'esprit : «Il ne reste que peu de gens de l'espèce dont il provient - il préférait le profit commun à son avantage ou à son plaisir $»^{42}$.

Trois autres occurrences apparaissent dans la seconde partie du texte, le miroir proprement dit, dans le cadre de la discussion sur la largesse et la cupidité. Deux d'entre elles concernent précisément cette dernière et résume ses effets dévastateurs :

What causid hir inward werre and rumour But avarice? Shee refte hem hir wele. Whyles they hadde in cheertee and favour Profyt commun, they hadden by the stele Prosperitee; but it away gan stele Whan they hem drow to profyt singuler, And of profyt commun nat weren cheer.

\begin{abstract}
Qu'est-ce qui a provoqué [à Rome] la guerre civile et les troubles, si ce n'est la cupidité ? Tant qu'ils favorisaient et étaient attirés par le bien commun, ils connaissaient la prospérité ; mais elle s'en est allée quand ils furent attirés par un intérêt particulier et qu'ils n'eurent plus d'attirance pour le bien commun.
\end{abstract}

vers $5244-5250$

La cupidité est donc mise en relation avec la guerre civile ce qui n'est évidemment pas innocent, dans le contexte dans lequel écrit Hoccleve, celui d'une période encore troublée par le changement de dynastie suite à la déposition de Richard II Plantagenet et à l'usurpation d'Henry IV de Lancastre en 1399, mais aussi dans le contexte de ce que les historiens anglais ont appelé le bastard feudalism, caractérisé par l'importance de l'argent dans les relations contractuelles ${ }^{43}$. Le poète travaille certes à la légitimation des Lancastres, mais on sent là comme un avertissement: s'ils veulent éviter le sort des Plantagenets, ils doivent cultiver la vertu de largesse et éviter à tout prix que ne se répandent les méfaits de la cupidité...

Si l'expression du bien commun - essentiellement sous sa forme commune profit - est relativement rare dans ce corpus de poésie anglaise, elle apparaît cependant toujours de manière très significative par rapport aux préoccupations des poètes, et toujours en relation avec le gouvernement du roi. Cela est évident chez Langland et Gower, mais aussi chez Ashby et chez les auteurs de Mum and the Sothsegger et des poèmes du manuscrit Digby 102, qui utilisent l'expression en relation avec une réflexion sur la question des limites du gouvernement du roi,

\footnotetext{
${ }^{41}$ Voir A. Mairey, Une Angleterre entre rêve et réalité, op. cit., p. 321-325. Sur la mode, voir S. Newton, Fashion in the Age of the Black Prince: A Study of the Years 1340-65, Woodbridge, 1980.

${ }^{42}$ Vers 1146-1148: O fewe been ther now left of the brood/ That he cam of - he loved bet profyt/ Commun than his avantage or delyt.

${ }^{43}$ Voir J.-P. Genet, La genèse de l'Etat moderne, op. cit., passim.
} 
que celles-ci soient positives - dans les discussions sur le conseil - ou plus restrictives, et l'exemple de Lycurgue dans la Confessio amantis est à cet égard très significatif. Dans ce cadre, le bien commun apparaît avant tout comme une notion générale concernant le bien-être - pas seulement matériel - de la communauté, en tant qu'objectif par excellence de la conduite d'un bon gouvernement. Il est cependant difficile de préciser si common profit relève plus précisément du bonum commune ou de l'utilitas commune...

Quant à la mise en relation, essentiellement par Hoccleve, du bien commun et de la trilogie largesse/prodigalité/cupidité, elle renvoie sans doute à une conception plus matérielle de la notion, mais cela ne diminue pas son importance ; surtout, le lien avec le gouvernement du roi est très net, dans la mesure où l'exercice de la largesse par le roi (avec en corollaire le contrôle de la cupidité des membres de la société) est un facteur de paix essentiel, dans une société où les relations contractuelles sont de plus en plus marquées par les questions monétaires. Une fois encore, l'utilisation d'une notion fréquemment releguée au rang de stéréotype s'avère complexe et lourde de sens dans la tentative de construction d'un dialogue entre le roi et ses sujets. 